\title{
3D Reconstruction of Coronary Stents in Vivo Based on Motion Compensated X-Ray Angiograms
}

\author{
Babak Movassaghi ${ }^{1,3}$, Dirk Schaefer ${ }^{2}$, Michael Grass $^{2}$, \\ Volker Rasche ${ }^{2}$, Onno Wink ${ }^{1,3}$, Joel A. Garcia ${ }^{3}$, James Y. Chen ${ }^{3}$, \\ John C. Messenger ${ }^{3}$, and John D. Carroll ${ }^{3}$ \\ ${ }^{1}$ Philips Research North America, 345 Scarborough Road, Briarcliff Manor, New York, USA \\ babak.movassaghi@philips.com \\ 2 Philips Research Laboratories, Sector Technical Systems Hamburg, Roentgenstrasse 24-26, \\ D-22335 Hamburg, Germany \\ ${ }^{3}$ University of Colorado Health Sciences Center Division of cardiology, 4200 East Ninth \\ Avenue, Denver, Colorado, USA
}

\begin{abstract}
A new method is introduce for the three-dimensional (3D) reconstruction of the coronary stents in-vivo utilizing two-dimensional projection images acquired during rotational angiography (RA). The method is based on the application of motion compensated techniques to the acquired angiograms resulting in a temporal snapshot of the stent within the cardiac cycle. For the first time results of 3D reconstructed coronary stents in vivo, with high spatial resolution are presented. The proposed method allows for a comprehensive and unique quantitative 3D assessment of stent expansion that rivals current $\mathrm{x}$-ray and intravascular ultrasound techniques.
\end{abstract}

\section{Introduction}

Stent deployment is currently the preferred vascular interventional procedure for stenosis treatment. During a percutaneous transluminal coronary angioplasty (PTCA) procedure a stent is delivered to the stenotic segment of a coronary artery using a ballontipped delivery catheter. The stent is crimped on the deflated balloon before insertion into the patient. The balloon catheter has two metallic markers with high absorption coefficients, which help the cardiologist to locate the balloon position in the angiograms. Once in place, the balloon is inflated, expanding the stent which locks in place and forms a scaffold. The complete expansion of the stent is essential to treating the stenosis. Inadequate expansion of the stent is a major predisposing factor to acute thrombosis, the formation of a blood clot within the stent resulting in loss of flow and an acute myocadial infarction, i.e. heart attack. Stents are positioned and deployed by fluoroscopic guidance. Although the current generation of stents are made of materials with some degree of radiopacity to enable detecting their location after deployment proper stent expansion is hard to asses. A considerable amount of work has been performed in order to visualize the coronary stent for improved positioning and outcome control, utilizing different non-invasive [11233,45] and invasive [617] imaging modalities. The main limitation of existing imaging methods is the limited spatial resolution, mainly caused due to cardiac and respiratory motion. While the future trend is heading towards noninvasive 
modalities for diagnosis and post-operative follow-up, there remains an interest to have a 3D representation of the deployed stent during a PTCA intervention using the same imaging modality, i.e. X-ray, as used for the stent deployment. The main challenge for constructing this $3 \mathrm{D}$ representation is the presence of cardiac and respiratory motion. One possible solution to derive more accurate images from moving objects such as the heart is to apply motion compensation techniques to the acquired data [8 9 10 $11 \mid 12$ |13]. In this paper an approach to accomplish a three-dimensional reconstruction of coronary stents in vivo is presented. In this approach the presence of markers on the balloon catheter is used to accurately determine their position, thus facilitating motion compensation. A reconstruction with high spatial resolution is achieved, by involving additional motion compensated projections in the reconstruction.

The paper is organized as follows: In Section 2 the method for accomplishing motion compensated 3D reconstruction of coronary stents is introduced. In Section 3, initial results on the application of the proposed method in a clinical settings are presented. Conclusions and suggestions for future work are given in Section 4

\section{Method}

In this section the methodology to derive high-resolution $3 \mathrm{D}$ reconstructed stents invivo is presented. The method described in this work is based on the assumption that the deployed stent has a negligible relative movement to the enclosed part of the guide wire. Therefore, the extraction of the motion of the stent is based on determining the motion of the markers on the balloon catheter. First, the data acquisition protocol is introduced. Second, the image processing methods for determining the position of the two markers is discussed. Third, it is described how the principles underlying coronary modeling can be utilized for generating an initial 3D representation of the two bullets in space. Finally, a 2D transformation matrix for each projection image is determined, which can be used for motion compensated coronary stent reconstruction.

\subsection{Data Acquisition}

The projection data are obtained during a continuous rotation of a calibrated monoplane C-arm X-ray system (Philips Integris Allura, Philips Medical Systems, Best, The Netherlands). A propeller rotation starting at $60^{\circ} \mathrm{RAO}$ and ending at $120^{\circ} \mathrm{LAO}$ is used, which ensures sufficient angular coverage for volume reconstruction. The angiographic projections were acquired at a frame rate of 30 frames per second resulting in 180 projection images during a 6 second rotational run. During acquisition the ECG signal is recorded in order to retrospectively determine projections acquired in the same cardiac phase. All data sets were acquired with a 512x512 image matrix.

\subsection{D Marker Extraction}

Figure 1 illustrates a typical X-ray angiogram, which is utilized to ensure correct deployment and positioning of the stent (inspection of the borders) acquired immediately after stent deployment in a routine interventional procedure. In such images, the balloon 


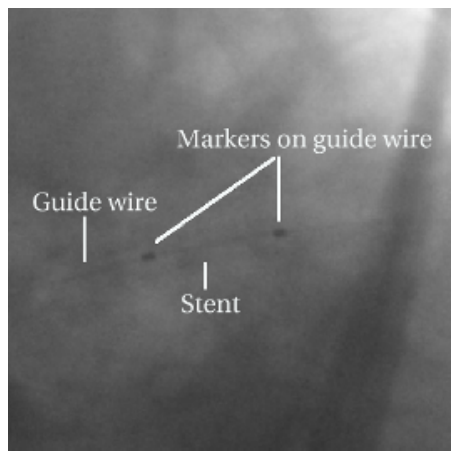

Fig. 1. X-ray angiogram, acquired immediately after a stent deployment. The markers can clearly be differentiated due to the high absorption coefficient.

markers can clearly be differentiated due to the high absorption coefficient. The guide wire, with smaller size, can also be identified. However, the deployed stent surrounding the guide wire in between the markers is barely visible.

The marker positions in all available projections are automatically extracted based on a segmentation algorithm, where the sub-pixel precise identification of the bullet center point is achieved using a coarse to fine approach utilizing scale space techniques as described in [14]15]. For each marker $\left(M_{1}^{i}, M_{2}{ }_{2}\right)$, this step yields a $2 \mathrm{D}$ position $(x, y)^{i}$, which depends on the projection number $i$.

\subsection{Reference 3D Marker Position}

Based on the ECG signal, two projection images are automatically selected belonging to the same cardiac phase where cardiac phases in the end-systole or late-diastole are preferred. In order to reconstruct a point $P^{3 D} \in R^{3}$ in space from two projections, first its corresponding image points $P_{A} \in R^{2}$ and $P_{B} \in R^{2}$ in the projections must be determined, as shown in Figure 2 (a). With the knowledge of the exact projections parameters including the location and orientation of the two focal spots $F_{A}$ and $F_{B}$ and the detector locations, the correspondence can be solved using the epipolar constraint [16]. The point $P_{B}$ is determined by searching only along the epipolar-line in projection $B$. The spatial position $P_{3 D}$ is computed by a simple intersection of the lines from the points on the projections to their respective focal points. If the points $P_{A} \in R^{2}$ and $P_{B} \in R^{2}$ are acquired for different motion states, the connecting lines from the points on the projections to their respective focal spot may not intersect (see Figure 2(b)). Nevertheless, the approximate 3D position can be determined by calculating the midpoint of the line with the smallest Euclidean distance between the two connecting lines as illustrated in Figure 2(b).

\subsection{Forward Projection}

The 2D position corresponding to a $3 \mathrm{D}$ point $P_{3 D}$ in an arbitrary projection $C$ can be determined by the forward projection of $P_{3 D}$ onto the projection plane $C$. The forward 


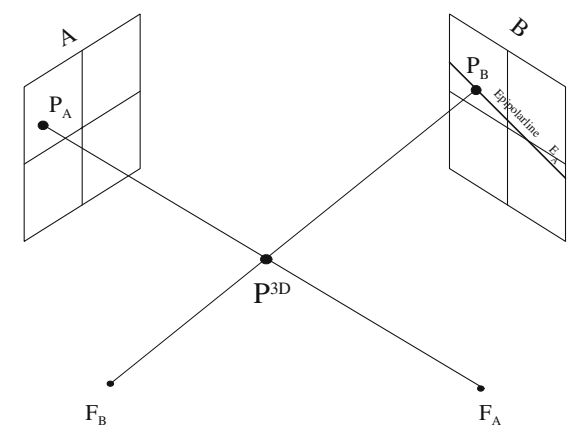

(a)

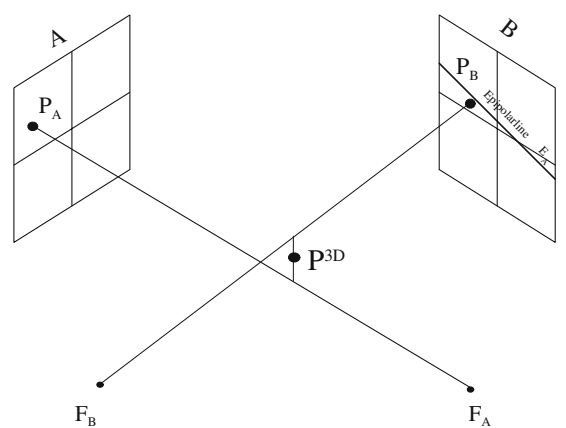

(b)

Fig. 2. Illustration of the $3 \mathrm{D}$ modeling technique in order to reconstruct a point $P^{3 D} \in R^{3}$ in space from two projections using the epipolar constraint. If the points $P_{A} \in R^{2}$ and $P_{B} \in R^{2}$ are acquired in the same motion state the connecting lines from the points on the projections to their respective focal spot intersect (a). If the points $P_{A}$ and $P_{B}$ are acquired for different motion states, the connecting lines may not intersect (b) and the $3 \mathrm{D}$ position can be approximated by calculating the midpoint of the line with minimum Euclidean distance between the two connecting lines (b).

projection of a $3 D$ location $P_{3 D}$, for a given orientation $(\alpha)$ of the C-arm, onto the projection plane $C$ at the point $P_{C}=\left(x_{c} ; y_{c}\right)$ is described by the projection matrix $M_{\alpha}$, according to:

$$
\left(\begin{array}{l}
x_{1} \\
x_{2} \\
x_{3}
\end{array}\right)=M_{\alpha}\left(\begin{array}{c}
P_{x} \\
P_{y} \\
P_{z} \\
1
\end{array}\right) \quad \text { with } \begin{aligned}
& x_{c}=x_{1} / x_{3} \\
& y_{c}=x_{2} / x_{3}
\end{aligned}
$$

In our application, the feature points (markers) are subject to periodic movement due to cardiac and respiratory motion. Every projection is acquired at a different projection geometry $\left(G_{i}\right)$ and belongs to a specific cardiac motion state $\left(T_{m}\right)$.

\subsection{D Motion Compensated Stent Reconstruction}

In this section we will introduce the methods for determining the motion owing to the coronary arteries, so as to compensate for this motion in projection images acquired in a different heart phase $T_{c_{0}}$. Hereto, the $2 \mathrm{D}$ position of the two balloon markers are determined in each projection such as described in Section 2.2. Based on the two images a 3D model of the two markers is modelled such as explained in Section 2.3. The two 3D markers are then forward projected into the acquired projections with different projection geometry and different motion state. Therefore, in each projection two positions are determined for every marker: the actual position for this specific projection geometry $\left(G_{i}\right)$ and cardiac motion state $\left(T_{m}\right)$, and a calculated position of the marker at this projection geometry $\left(G_{i}\right)$ but at the cardiac motion state $T_{c_{0}}$ (see Figure 3). All projections are then motion compensated into cardiac motion state $T_{c_{0}}$, by determining the transformation that brings the actual position of the marker in correspondence with the calculated position. In this initial study a affine transformation is applied. All 


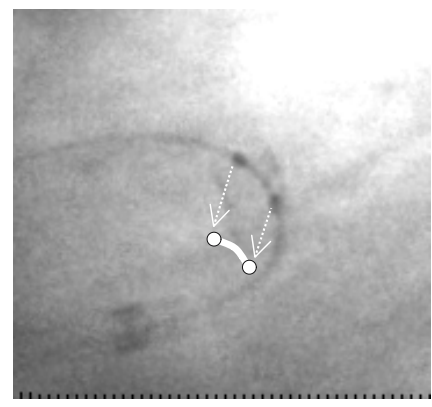

(a)

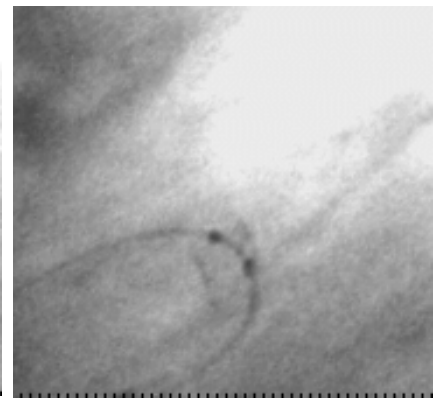

(b)

Fig. 3. A projection acquired at motion state $T_{m}$ before (a) and after warping (b) into the optimal cardiac phase $\left(T_{c_{0}}\right)$ based on a Thin Plate Spline (TPS) transformation matrix $(\Phi)$

corresponding points such as reference marker are warped exactly. The other pixels in each slice are warped linear depending on the distance to the landmarks. In Figure 3 . a projection is shown for a motion state $T_{m} 3(\mathrm{a})$ and its warped projection 3 (b), transformed into cardiac phase $\left(T_{c_{0}}\right)$. Using this approach all projection images are motion compensated into cardiac phase $T_{c_{0}}$. These projection are then used as an input for a standard 3D filtered back-projection reconstruction algorithm such as Feldkamp [17].

\subsection{Initial Experiments on Patient Data}

In this work we present initial results of patient data sets of an ongoing study carried out at the Division of Cardiology at the University of Colorado Health Science Center (UCHSC). The objective of the study is to investigate the feasibility of fully automated $3 \mathrm{D}$ reconstruction of deployed coronary stents and other intra-cardiac devices in-vivo to enable device assessment and monitoring immediately after deployment and longterm. Angiograms are selected for inclusion in this study from patients who undergo coronary stent deployment in the proximal or mid left anterior descending (LAD), the proximal or mid circumflex $(\mathrm{CX})$ artery, obtuse marginal branches $(\mathrm{OM})$, or the proximal, mid, or distal right coronary artery (RCA). Up till now, data sets from 11 patients with intracoronary stents $\left(\right.$ CYPHER $^{\circledR}$ Sirolimus-eluting Coronary Stent) were acquired. Depending on the size of the respective stenosis, three different stent sizes were used: the diameter of the stents were either $2.75 \mathrm{~mm}$ or $3.0 \mathrm{~mm}$ and the stent length (unexpanded) varied between $8 \mathrm{~mm}$ and $23 \mathrm{~mm}$. The acquisition protocol was carried out such as explained in Section 2.1. The acquisition was performed directly after stent implementation. The heart rate of the patients varied between 52 and 71 beats per minute. Since the center of the heart is not on the central axis of the patient, but located within the left thorax, the patients had to be positioned off-iso-center to ensure proper alignment of the iso-center of the system with the center of the heart. The diameter of the image intensifier was preferably chosen at 5 inch. However, the acquisition of large patients was carried out at 7 inch assuring the correct iso-centering of the system with the center of the markers. 


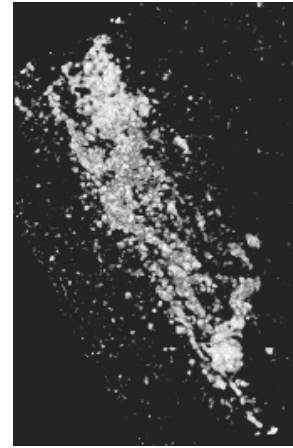

(a)

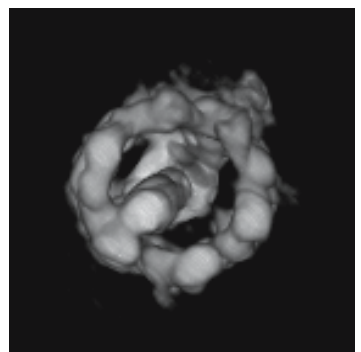

(c)

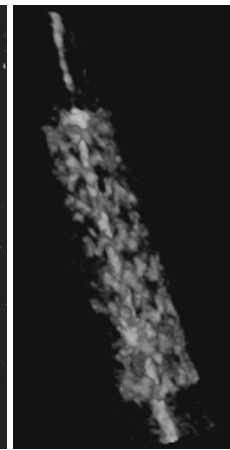

(b)

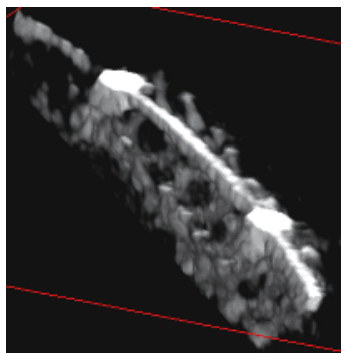

(d)

Fig. 4. Volume rendered images of a 3D reconstructed stent deployed in patient one, utilizing the original data set (a) and utilizing motion compensated data set (b). The struts of the stent have a diameter of approximately $40 \mu \mathrm{m}$.C ut planes perpendicular (c) and parallel (d) to the longitudinal direction of the volume rendered images of the reconstructed stent are visualized revealing information about the in-stent lumen.

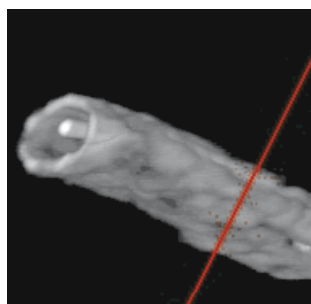

(a)

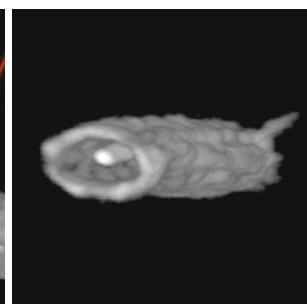

(b)

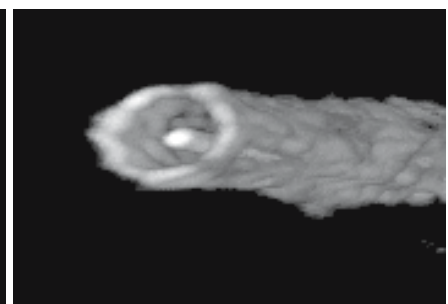

(c)

Fig. 5. Illustration of volume rendered images of a deployed stent in patient two. Different cut planes perpendicular to the tangential direction of the stent have been chosen to visualize the inner-stent lumen.

\section{Results}

In all 11 cases the deployed stent could be reconstruction successfully enabling high resolution images. The image quality varied based on the patient size and best results could be achieved in small patients $(<60 \mathrm{~kg})$. In Figure 4 exemplary images of a 3D 
reconstructed stent in-vivo, utilizing the original data set (Figure 4a)) and utilizing a motion compensated data set (Figure 4(b)) are presented. From Figure 4 b) it can be seen that the meshes $(\varnothing<114 \mu \mathrm{m})$ of the stent can be visualized, which shows that a very high spatial resolution is achieved. In Figures $4(\mathrm{c}, \mathrm{d})$ cut planes perpendicular(c) and parallel(d) to the longitudinal direction of the stent are visualized revealing information about the in-stent lumen. Volume rendered images of stents in different patients are illustrated in Figure 5. Different cut planes perpendicular to the tangential direction of the stent have been chosen to visualize the inner-stent lumen. Different viewing angles are selected to demonstrate the 3D aspect.

\section{Discussion}

A fully automatic method has been presented that produces a $3 \mathrm{D}$ reconstruction of coronary stents after deployment in patients. The proposed method achieves a high spatial resolution (up to $40 \mu \mathrm{m}$ ), by implementing additional projections that have been motion compensated in the reconstruction algorithm. The method was applied to 11 patient data sets and is subject of an ongoing study at the UCHSC. The coronary stents could be reconstructed and visualized in all cases. Best results were achieved utilizing the smallest image intensifier size ( $\varnothing$ 5inch).

The proposed method supports cardiologists in allowing an immediate assessment of the completeness of stent deployment and expansion during a PTCA procedure. This method of X-ray based automatic $3 \mathrm{D}$ reconstruction has the potential to replace the need for intravascular ultrasound. The 3D stent reconstruction can be used to identify which stents do and which do not need addtional high-pressure balloon inflations to optimize treatment of the stenosis and minimize the risk of stent thrombosis. Unlike intravascular ultrasound that requires addtional equipment and adds more complexicity to the procedure, $3 \mathrm{D}$ stent reconstruction uses the existing imaging system and will add minimal time to the procedure. Thus, the 3D stent reconstruction tool could provide a cost-effective and rapid assessment of stent deployment leading to improved patient outcomes. Clinical studies showing the economic and patient outcomes effects will be executed after further technical refinements. A limitation of the method is the need of the markers positions in each projection. Thus, the proposed method can only be carried out during a invasive procedure. Furthermore the proposed method is only accurate for stent segments enclosing the guide wire segment between the markers. In future work more accurate results might be achieved by 1) incorporation of the guide wire between the two markers. 2) determination of the 4D motion vector field of the markers and guide wire in space; 3 ) incorporation of markers into the body of the stent itself obviating the need of using the guide wire and delivery balloon markers.

\section{References}

1. K. Nieman, F. Cademartiri, R. Raaijmakers, P. Pattynama, and P. de Feyter, "Noninvasive angiographic evaluation of coronary stents with multi-slice spiral computed tomography," Herz, vol. 28, no. 2, pp. $136-42,2003$.

2. K. Nieman, J. M.R. Ligthart, P. W. Serruys, and P. J. de Feyter, "Left main rapamycin-coated stent: Invasive versus noninvasive angiographic follow-up," Circulation, vol. 105, pp. 130 $131,2002$. 
3. N. Funabashi, N. Komiyama, and I. Komuro, "Patency of coronary artery lumen surrounded by metallic stent evaluated by three dimensional volume rendering images using ecg gated multislice computed tomography," Heart, vol. 89, pp. 388, 2003.

4. D. Maintz, KU. Juergens, T. Wichter, M. Grude, W. Heindel, and R. Fischbach, "Imaging of coronary artery stents using multislice computed tomography: in vitro evaluation," Eur Radiol., vol. 13, no. 4, pp. 830-835, 2003.

5. D. Maintz, H. Seifarth, T. Flohr, S. Kramer, T. Wichter, W. Heindel, and R. Fischbach, "Improved coronary artery stent visualization and in-stent stenosis detection using 16-slice computed-tomography and dedicated image reconstruction technique," Invest Radiol., vol. 38, no. 12, pp. 790-795, 2003.

6. Degertekin M Ligthart JM Oortman RM Serruys PW Slager CJ Tanabe K, Gijsen FJ, "Images in cardiovascular medicine. true three-dimensional reconstructed images showing lumen enlargement after sirolimus-eluting stent implantation," Circulation, vol. 106, no. 22, pp. e179-180, 2002.

7. P. Delachartre, E. Brusseau, F. Guerault, G. Finet, C. Cachard, and D. Vray, "Correction of geometric artifacts in intravascular ultrasound imaging during stent implantation," IEEE International Ultrasonics Symposium,Caesars Tahoe, Nevada, 1999.

8. B. Movassaghi, V. Rasche, M. A. Viergever, and W. J. Niessen, "3D coronary reconstruction from calibrated motion-compensated 2D projections based on semi-automated feature point detection," in Proc. SPIE Medical Imaging: Image Processing, San Diego, CA, 2004, vol. 5370, pp. 1943-1950.

9. C. Blondel, R. Vaillant, G. Malandain, and N. Ayache, "3-d tomographic reconstruction of coronary arteries using a precomputed 4-d motion field.," Physics in Medicine and Biology, vol. 49, no. 11, pp. 2197-2208, 2004.

10. G. Shechter, F. Devernay, E. Coste-Maniere, A. Quyyumi, and E.R. McVeigh, "Threedimensional motion tracking of coronary arteries in biplane cineangiograms," IEEE Trans. Med. Imag., pp. 493-503, 2003.

11. M. Moriyama, Y. Sato, H. Naito, M. Hanayama, T. Ueguchi, T. Harada, F. Yoshimoto, and S. Tamura, "Reconstruction of time-varying 3-d left-ventricular shape from multiview X-ray cineangiocardiograms," IEEE Trans. Med. Imag., vol. 21, no. 7, pp. 773-785, 2002.

12. C. Blondel, R. Vaillant, F. Devernay, G. Malandain, and N. Ayache, "Automatic trinocular $3 \mathrm{~d}$ reconstruction of coronary artery centerlines from rotational x-ray angiography," in Computer Assisted Radiology and Surgery 2002 Proceedings (CAR '02). 2002, pp. 832 - 837, Springer.

13. B. Movassaghi, V. Rasche, R. Florent, M. A. Viergever, and W. J. Niessen, "3D coronary reconstruction from calibrated motion-compensated 2D projections," in Computer Assisted Radiology and Surgery (CARS 2003). 2003, pp. 1079-1084, Elsevier.

14. T. Netsch and H. O. Peitgen, "Scale-space signatures for the detection of clustered microcalcifications in digital mammograms," IEEE Trans. Med. Imag., vol. 18, no. 9, pp. 774 - 786, 1999.

15. T. Netsch, "A scale-space approach for the detection of clustered microcalcifications in digital mammograms," Digital Mammography '96, pp. 301 -306, 1996.

16. T.J. Keating, P.R. Wolf, and F.L. Scarpace, "An improved method of digital image correlation," Photogrammetric Eng. Remote Sensing, vol. 41, pp. 993 - 1002, 1975.

17. M. Grass, R. Koppe, E. Klotz, R. Proksa, M.H. Kuhn, H. Aerts, J. Op de Beek, and R. Kemkers, "3D reconstruction of high contrast objects using C-arm image intensifer projection data," Computer. Med. Imaging Graphics, vol. 23, no. 6, pp. 311 - 321, 1999. 